\title{
Motion Biomarkers for Early Detection of Dementia-Related Agitation
}

\author{
Ridwan Alam ${ }^{1}$, Jiaqi Gong ${ }^{1}$, Mark Hanson ${ }^{2}$, \\ Azziza Bankole ${ }^{3}$, Martha Anderson ${ }^{4}$, Tonya Smith-Jackson ${ }^{5}$, John Lach ${ }^{1}$ \\ ${ }^{1}$ Department of Electrical and Computer Engineering, University of Virginia, Charlottesville, VA \\ ${ }^{2}$ School of Medicine and Health Sciences, George Washington University, Washington, DC \\ ${ }^{3}$ Department of Psychiatry \& Behavioral Medicine, Virginia Tech Carilion School of Medicine, Roanoke, VA \\ ${ }^{4}$ Department of Interprofessionalism, Virginia Tech Carilion School of Medicine, Roanoke, VA \\ ${ }^{5}$ Department of Industrial \& System Engineering, North Carolina A\&T State University, Greensboro, NC \\ Email: ridwan@virginia.edu, jlach@virginia.edu
}

\begin{abstract}
Agitation in dementia poses a major health risk for both the patients and their caregivers and induces a huge caregiving burden. Early detection of agitation can facilitate timely intervention and prevent escalation of critical episodes. Sensing behavioral patterns for detecting health critical events is a challenging task. Wearable sensors are often employed for sensing physiological signals, but extracting possible biomarkers for confident detection of early agitation is still an open research. In this paper, we employ an ongoing iterative study to explore the motion biomarkers related to agitation in community-dwelling persons with dementia (PWD). This study uses accelerometers in smart watches to capture PWD behavioral patterns unobtrusively. Analysis of the feature space is performed using data from multiple subjects to discriminate among epochs of onset, preset, and offset of agitation while considering inter-person variability in real deployments. This paper shows the prospect of feature space analysis of the motion data for developing early agitation detection models to deploy in the wild.
\end{abstract}

\section{Keywords}

Wearable; Dementia; Agitation; Accelerometer; Effect Size; PCA; K-Means Clustering; Early Detection.

\section{INTRODUCTION}

Dementia is a chronic and progressive neurodegenerative disorder. $60-80 \%$ cases of dementia are Alzheimer's dementia. There are 5.4 million people in the US with a diagnosis of such dementia. $11 \%$ Americans who are more than 65 years old and $32 \%$ people 85 years or older have Alzheimer's disease [1]. Dementia patients suffer from memory loss, cognitive, visual, and vocal impairments. These symptoms progressively worsen over time, and patients often express agitated behaviors. Of the 15.9 million Americans

This project is supported in part by the National Science Foundation under grant IIS-1418622.

Permission to make digital or hard copies of all or part of this work for personal or classroom use is granted without fee provided that copies are not made or distributed for profit or commercial advantage and that copies bear this notice and the full citation on the first page. Copyrights for components of this work owned by others than ACM must be honored. Abstracting with credit is permitted. To copy otherwise, or republish, to post on servers or to redistribute to lists, requires prior specific permission and/or a fee. Request permissions from Permissions@acm.org. DigitalBioMarkers'17, June 23, 2017, Niagara Falls, NY, USA

(C) 2017 Association for Computing Machinery.

ACM ISBN 978-1-4503-4963-5/17/06.. \$15.00

http://dx.doi.org/10.1145/3089341.3089344 who provide unpaid dementia care, the majority is family members and friends. However, caring for a person with dementia (PWD) is physically and emotionally taxing for caregivers. A critical stressor for caregivers is the agitation episodes in PWD. Agitation occurs in up to $90 \%$ of PWD [2] and is one of the principal factors prompting institutionalization of community-dwelling PWD [3]. As the number of dementia patients is projected to rise to 13 million by 2050, alternate solutions of institutionalization like aging-in-place are highly encouraged to reduce both government and personal cost burden $[1,3]$. Toward that goal, reducing caregiver burden and increasing their self-efficacy is a major challenge.

Agitation in dementia is described as a set of either repetitious behaviors like pacing, verbal repetition, etc. or socially inappropriate and aggressive behaviors such as verbal outbursts or hitting. Most agitation interventions are reactive rather than proactive and are administered too late in an agitation escalation to be routinely effective [4]. Early detection of agitation is considered clinically significant and promotes intervention, which may reduce the risk of possible harm to the individual or others.

State-of-the-art sensing and monitoring systems aimed toward dementia care focus on detecting early stages of the disease, providing support for cognitive care, and therapy or internvention mechanisms $[5,6]$. Continuous and real-time detection, prediction, and prevention of severe agitation episodes is still unexplored. Available technologies are often privacy invasive or less feasible for residential long term deployments [6-8]. Wearable inertial sensors has shown great potential for monitoring behavioral and physiological parameters non-invasively and unobtrusively.

Application of inertial sensors for behavioral pattern detection faces manifold challenges including confounding activities and gestures $[6,8]$, inter-person variability in physical motion patterns, lack of reliable and accurate ground truth [8], inter-rater variability among caregivers, and real world deployment and compliance challenges. We attempt to address these issues as part of an ongoing study on Behavioral and Environmental Sensing and Intervention (BESI) for dementia caregiver empowerment [9]. In this work, we attempt to find reliable motion biomarkers for detecting early signs of agitation. In that endeavor, we conduct statistical analysis like effect size analysis, k-means clustering, and principal component analysis on motion data collected from real patients in residential settings. We explore the features from motion data to detect and predict agitation before escalation and to notify the caregiver with personalized interventions and prevent escalation.

In this paper, we describe our approaches for discriminating among non-agitation, pre-agitation, and agitation episodes. Related works in this area of research are briefly described in the next section. 
Section 3 describes the BESI study and the wearable system employed to collect motion data. The data processing and analysis methodology is explained in Section 4. Result from our exploration of digital motion biomarkers for agitation prediction is presented in Section 5 with a following discussion on future works.

\section{RELATED WORK}

The severity and stages of behavioral and psychological symptoms of dementia (BPSD) like agitation and aggressive behaviors are quantified using standardized clinical assessment tools $[2,4]$. One most widely used tool for assessing such behaviors is the CohenMansfield agitation inventory (CMAI) which attempts to measure levels of physically and verbally aggressive and non-aggressive behaviors [4]. While this tool assesses the agitation severity, there exists methods for quantifying overall progression of the disease. Clinical dementia rating (CDR) [10] evaluates patients' cognitive and functional performance to determine the stage of dementia. The modified mini-mental state (3MS) examination is an assessment of cognitive abilities of the patient including memory, recall, orientation, attention, and language use [11]. These tools are subjective inputs from caregivers or clinicians, yet they are still the most accepted gold standards for assessing dementia and agitation.

Sensing and monitoring technologies attempt to supplement the standardized clinical assessments using objective modalities and to provide multifaceted dementia care [5]. These dementia care include patient monitoring in clinical and residential setting, early diagnosis of the disease, assistive technologies for improving quality of life, and therapies and intervention [5-8]. Detecting cues of agitation escalation in diagnosed dementia patients emphasizes continuous monitoring of the behavioral parameters. State-of-theart approaches use varying modality of sensors [5,6] e.g. bodyworn motion, acoustic, physiological sensors (heart rate, skin response, etc.), in-home passive infra-red (PIR) motion detectors, video cameras, pressure pads, and smart assisted living systems. These systems detect daily activity patterns, changes in health status, room occupancy, or anomalous behavior [6,8]. Among these sensing modalities, wearable inertial sensors have emerged as an unobtrusive and non-invasive system for continuous monitoring and have been tested in clinical and residential setting [5-7].

Wearable inertial sensors like accelerometer and gyroscope measure physical motion parameters of the wearer. Such measurements collected from dementia patients with agitation symptoms were compared with CMAI scores to correlate between the objective sensor data and the subjective clinical assessment scores [12]. This work focused on correlating the CMAI scores with the overall daily activity level captured by the wrist-worn sensor with an aim to supplement diagnosis of the disease. Similar approaches of measuring overall activity level were taken in [13] to demonstrate detection accuracy of treatment outcomes for dementia patients with agitation symptoms. They used wrist-worn actigraphic sensor data collected for 14 days to discriminate between healthy controls and dementia patients and compared those outcomes with NPI scores. Relationship between overall activity levels over certain periods and clinical assessment scales for other symptoms of dementia like apathy were also analyzed [14]. Analysis of ankle-worn accelerometer data from healthy controls and community-dwelling dementia patients showed prospect of early diagnosis as well as detecting changes in everyday behavior [15]. Though these works provide significant insight about the relationship between motion sensing and disease status, they do not provide confident biomarkers for detecting instantaneous escalation of agitation episodes in dementia patients.
Continuous monitoring of instantaneous changes in motion and activity using wearable sensors are performed for various health applications. One such application for elderly population is fall detection $[6,16]$ where motion data from body-worn sensors are analyzed for detecting abrupt changes in posture. Accelerometer data are also used to detect wandering outside residence for dementia patients through continuous monitoring [17]. Such applications utilize defined structures for the motion events like fall or wandering to design the detection strategy. On the other hand, agitation events are unpredictable both in occurrence and in nature as they contain both inter-event and inter-person variability. In an earlier study, we demonstrated the potential of instantaneous inertial motion signals to correlate with agitation events of dementia patients in controlled clinical settings [18]. A custom wrist-worn device (TEMPO [19]) was used to collect motion data from dementia patients in long-time care facilities while a trained clinical researcher monitored and collected ground truth. Teager analysis based detection algorithm was developed and validated using clinical measures like CMAI and the Aggressive Behavior Scale (ABS) [18]. Leveraging those findings, the current BESI study aims to detect, predict, and prevent escalation of agitation episodes in real-time for PWD in residential settings [9].

\section{BESI STUDY}

BESI is an ongoing study designed for a 3-phase, 4-year iterative exploration using descriptive, qualitative, and quantitative measures of caregiver-PWD dyads. Phase 1 of the study was focused on system development and verification, which yielded and validated the reliable BESI system [9]. Phase 2 is currently advancing with an aim to develop algorithms for early agitation detection and to model the behavioral patterns. And Phase 3 will incorporate early detection based just-in-time interventions to prevent escalation of critical agitation events and thus provide caregiver empowerment and reduce caregiving burden.

\subsection{Recruitment and Sample Size}

Dyads with a past or current participation in dementia support groups and community advocacy groups are recruited following a protocol approved by the IRB at Carilion School of Medicine. In Phase 1, two 1-week deployments in the community setting were
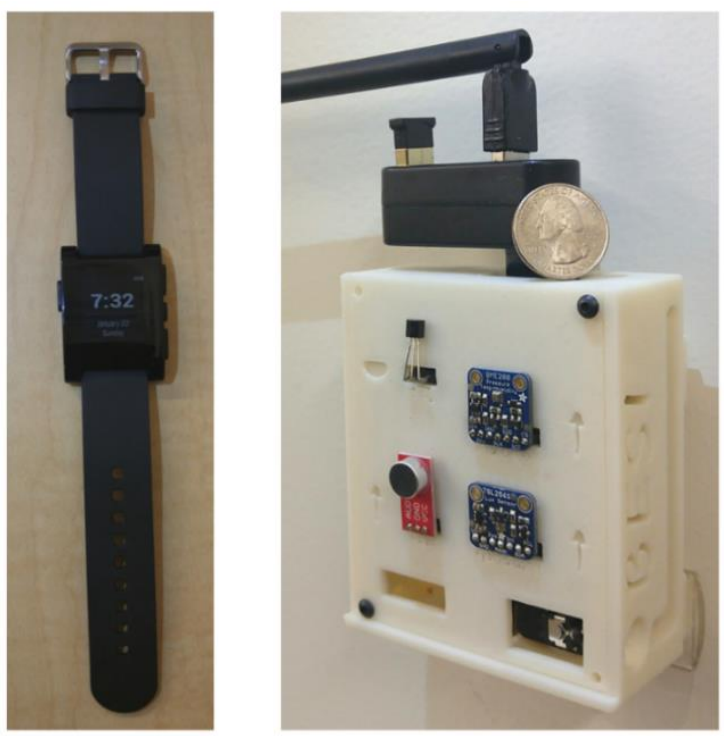

Figure 1. The Pebble watch and a room-level node of BESI [9] 
completed for system validation. In Phase 2, BESI is deployed for 30 days in the residence of each dyad, and we are validating the effectiveness of the collected data in assessing agitation. In Phase 2 the target sample size is eight dyads for 30 days each, and in Phase 3 the target sample size will be 12 dyads for 60 days each. We have already completed data collection from three dyads in Phase 2 and those data are used in this work.

\subsection{Data Collection}

The data collection pipeline in the BESI system consists of three components: a wearable sensing device, room-level nodes for data storage and processing, and a tablet based app for collecting ground truth observations about agitation events and other activities.

Motion data sensing from the PWD is performed by employing a wrist-worn accelerometer sensor. Considering ergonomic and compliance requirements, we use a Pebble smart watch as the wearable in BESI (Figure 1). The Pebble smart watch features an ARM Cortex-M3 processor and a peripheral 3-axis accelerometer with programmable sampling rates of 25,50 , or $100 \mathrm{~Hz}$. To collect the inertial motion data of the PWD, we developed a background worker named Pixie. The Pixie worker samples the accelerometer at $50 \mathrm{~Hz}$ and sends that data to any Bluetooth 4.0 (BT) connected room-level nodes in batches at power efficient time intervals using the data logging API. The Pixie worker incorporates a companion foreground app that displays a standard watch-face and handles accidental button presses by the PWD.

The room-level nodes are part of the BESI distributed system and performs a spectrum of functions (Figure 1). For collecting the motion data, these nodes are programmed against the constraint of constantly carrying a phone by the elderly PWD. These nodes connect with the Pebble watch over BT and receive motion data. We developed processes for each node to maintain continuous connection and data link by utilizing an open source python library Libpebble2. This library provides validated implementations of several Pebble protocols including data logging. This protocol enables receiving sampled accelerometer data from the watch in a reliable and efficient way. Data storage is handled on the nodes by incorporating the ZeroMQ library. The developed processes run in parallel on the nodes and store data on a mounted SD card.

An android app is developed to facilitate a daily survey on a tablet device. This app is used by the caregiver to provide observations about the agitation episodes including approximate time and location of occurrence, the caregivers perceived severity level (PSL) of the episode, and the physical and verbal symptoms expressed during the episode (e.g. pacing, hitting, repetition, shouting, etc.) as well as their daily activities and events.

\section{METHODOLOGY}

Sensor and survey data from the deployments are used here with a goal to detect the motion markers of early agitation. The demographic information of those PWDs along with some related clinical scores about the stages of their disease conditions are presented in Table 1. The number of agitation episodes along with the average PSL scores for those episodes as reported by the caregiver are also included.

\subsection{Data Pre-processing}

The data preprocessing steps include median filter based noise and artifact reduction and short duration lost data imputation. Data lost for long periods due to moving out of the coverage area are not considered for imputation. Short bursts of data loss for a few
Table 1. Demographic and Health Information

\begin{tabular}{|l|l|l|l|}
\hline Patient ID & PWD\#1 & PWD\#2 & PWD\#3 \\
\hline Age, Sex & $80, \mathrm{~F}$ & $69, \mathrm{M}$ & $82, \mathrm{M}$ \\
\hline CDR & $1.5 / 3$ & $2 / 3$ & $1.42 / 3$ \\
\hline 3MS & $59 / 100$ & $23 / 100$ & $50 / 100$ \\
\hline CMAI-C & $35 / 259$ & $49 / 259$ & $73 / 259$ \\
\hline \# of Agitation & 6 & 12 & 22 \\
\hline Avg PSL & $1.43 / 10$ & $3.61 / 10$ & $6.47 / 10$ \\
\hline
\end{tabular}

minutes due to connection hand-over, momentary obstruction in data path, or sensing hardware issues are imputed. A Gaussian model is built using data from the previous hour and is used to generate missing data for those few minutes. These imputed data are used for epoch based feature extraction and further analysis.

\subsection{Feature Extraction}

The first step for extracting features is to determine the epochs of interest (EoI). As pointed out in our earlier work [18], the agitation epochs vary in length even for the same patient. In that work, manually extracted 2-minute epoch was used to measure signal energy for each EoI. Since we want to explore all possible feature spaces in this work, we have empirically defined 10-minute epochs of motion data as representative for an EoI.

With a goal to detect early signs of agitation, we focus on three categories of EoI: agitation, pre-agitation, and non-agitation. The epoch for an agitation event spreads from 5 minutes before the reported occurrence to 5 minutes after that marking. These both sided windows minimize the effects of reporting bias and inaccuracy in the caregiver inputs. The pre-agitation epoch spreads from 15 minute to 5 minute before the annotation. Since agitation events are highly sporadic and infrequent compared to the duration of the whole deployment, the data set becomes an imbalanced set of epochs. To handle this issue, we perform random undersampling of the majority class [20]. We randomly pick from the remaining time series data the same number of 10-minute epochs as there were agitation epochs. We use these epochs to evaluate the hypothesis that certain features can be used to discriminate the preset, onset, and offset of agitation.

To capture the temporal dynamics as well as the variation in the feature space, we use a 6 -second sliding window over the 10 minute epoch with a $50 \%$ overlap and extract features from those sliding windows. Since the accelerometer sampling rate was programmed to be $50 \mathrm{~Hz}$, each feature instance was extracted using 300 samples from a 6-second window. For a 10-minute epoch of any type, we had 199 instances over the feature space.

We calculate statistical, frequency, and energy features from each window for the 3-dimensional time series signal from the 3-axis accelerometer. Statistical features include mean, median, standard deviation, variance, root mean square level, skewness, kurtosis, interquartile range, and pairwise correlation between axes signals [21]. Frequency features include frequency and power spectrum density values for peaks in $0-5 \mathrm{~Hz}, 5-10 \mathrm{~Hz}$, and $>10 \mathrm{~Hz}$ ranges, zero crossing rate, and mean crossing rate. Mean and standard deviation of Teager energy are also calculated to represent the change in energy of the signal [18]. 21 features are extracted for each axis of the accelerometer signal. Hence a total of 199 instances with 63 features are used to characterize each pre-agitation, agitation, or non-agitation epoch. 


\subsection{Effect Size Analysis and Clustering}

To discriminate among pre-agitation, agitation, and non-agitation epochs, we first try to analyze the dynamics for each epoch over the feature space and compare those among different epoch categories. The dynamic variation over the feature space in an epoch is measured using a dissimilarity matrix. The dissimilarity matrix is calculated using $\mathrm{L}^{2}$ norm of the distances between each pair of instances for an epoch. This results in a symmetric matrix of size $199 \times 199$ elements for each epoch. After summarizing this matrix by its 2-D mean and standard deviation, the dynamics for each 10minute epoch are represented by these two metrics. We then conduct effect size analysis to discover the performance of these metrics for discriminating among different categories of epochs. We conducted the analysis on individual PWD epochs as well as on the combined set of all PWD. The overall analysis considers all pre-agitation, agitation, and non-agitation epochs ignoring individual variations, while the personalized analysis only considers the epochs for an individual participant.

To evaluate the potential of this feature space in categorizing the instances among pre-agitation, agitation, and non-agitation, we also perform unsupervised k-means clustering of the instances into 3 groups. The clustering operations are performed both on individual PWD level instance sets and on the combined instance set for all PWD. The performances of the unsupervised clustering operations are then evaluated using the known epoch categories for the instances. Sensitivity and specificity are used as metrics of evaluation for all categories of epochs.

\subsection{Principal Component Analysis}

With a 63-dimensional feature space, it is difficult to analytically describe the clusters while some features may introduce collinearity or overfitting. To get a better perspective of the feature space, we conduct principal component analysis (PCA) on the individual PWD epoch set as well as on the combined epoch set after standardization. The resulting principal components provide information about the variance spread of the features, i.e. the proportion of variance explained by each component. The components also show the features that are correlated with each other as well as with the PCs. We translated the feature space to PCA space by calculating the PC scores for all instances. This translation does not affect the interpretations as the variance of the original space is maintained in the component score space.

On the principal component score space, we conduct effect size analysis as before to observe its ability to discriminate among different categories of epochs in both individual and combined level. We then perform $\mathrm{k}$-means clustering on the PC scores of the instances to evaluate the unsupervised clusters alignment with the known categories. The outcomes of these analysis and evaluation are presented in the following section.

\section{RESULTS}

The effect-size analysis on the 63-dimensional feature space shows the differences in the dynamics among the non-agitation, preagitation, and agitation epochs. The result of the analysis using the metrics (mean and standard deviation) from the dissimilarity matrices of the epochs is presented in Table 2 and the distribution of those metrics are shown in Figures 2-5. In these figures, the distributions of the two metrics are shown across the three epoch categories for both individual and overall analyses. We see that the effect sizes using both metrics between non-agitation and agitation and between non-agitation and pre-agitation are high for PWD 1,
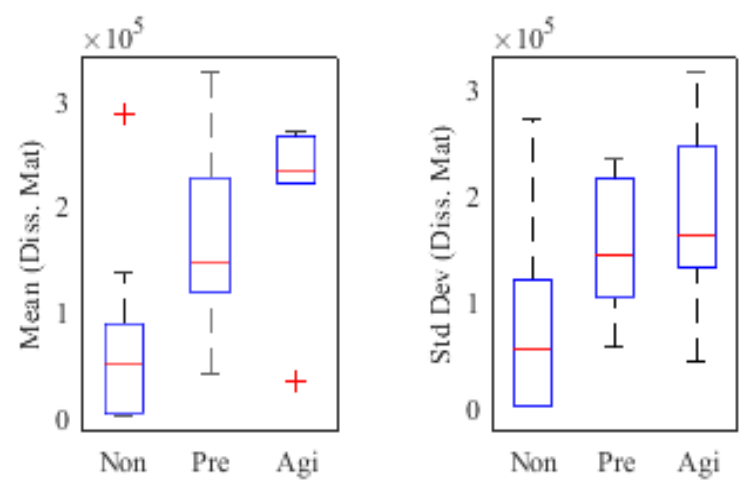

Figure 2. Distribution of mean and standard deviation of epoch features dissimilarity matrices for PWD 1.
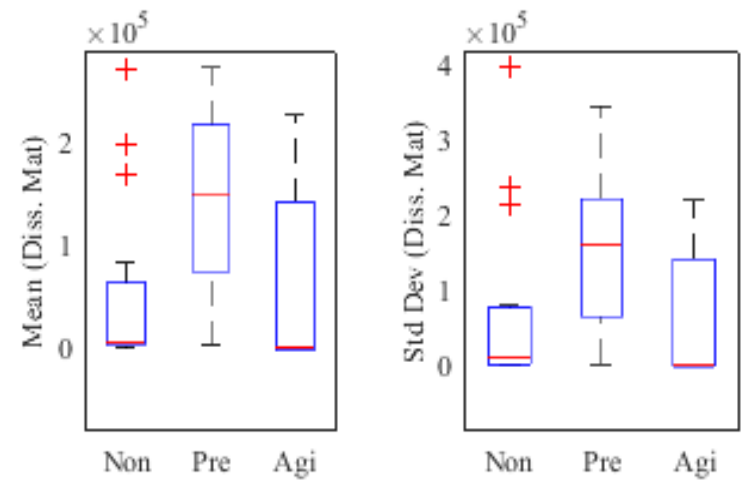

Figure 3. Distribution of mean and standard deviation of epoch features dissimilarity matrices for PWD 2.
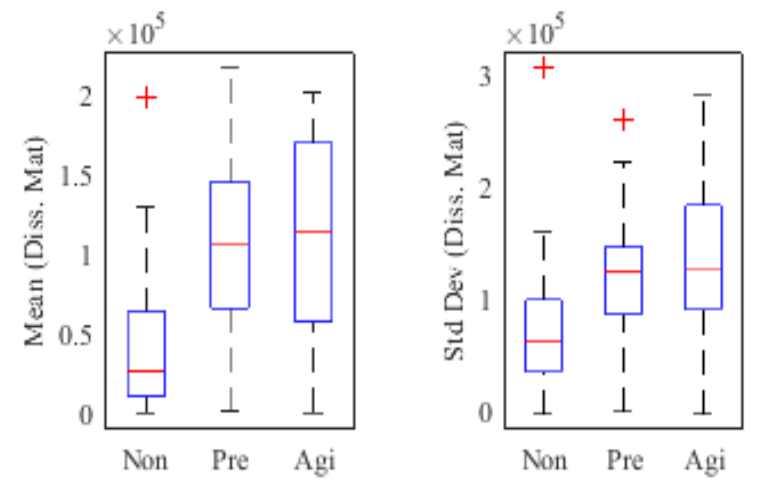

Figure 4. Distribution of mean and standard deviation of epoch features dissimilarity matrices for PWD 3.
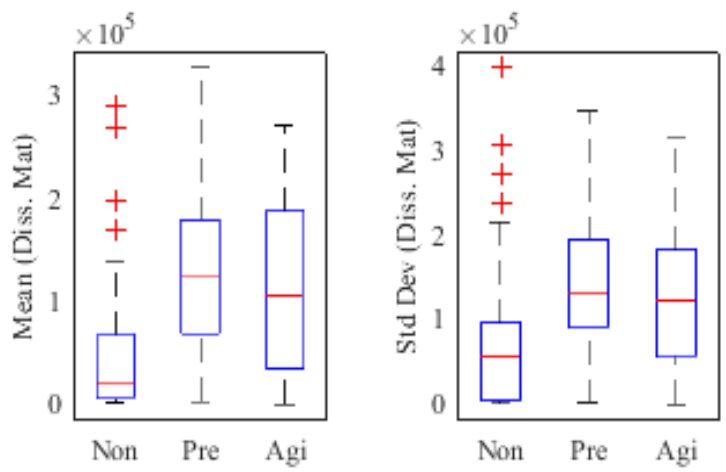

Figure 5. Distribution of mean and standard deviation of epoch features dissimilarity matrices for all PWDs. 
Table 2. Pairwise effect-size (ES) analysis of the mean and standard deviation of the epoch dissimilarity matrix among three categories: non-agitation $(\mathrm{N})$, pre-agitation $(\mathrm{P})$, and agitation (A) for individual PWD and all of them combined.

\begin{tabular}{|c|c|c|c|c|c|c|c|}
\hline \multirow{2}{*}{ Met. } & \multirow{2}{*}{$\begin{array}{c}\text { PWD } \\
\text { ID }\end{array}$} & \multicolumn{3}{|c|}{ ES } & \multicolumn{3}{|c|}{ PCA_ES } \\
\hline & & N-P & P-A & A-N & N-P & P-A & $\mathbf{A - N}$ \\
\hline \multirow{4}{*}{$\sum_{\Sigma}^{\bar{\Xi}}$} & 1 & 1.11 & 0.49 & 1.65 & 1.28 & 0.07 & 1.31 \\
\hline & 2 & 1.10 & 0.95 & 0.10 & 1.11 & 1.33 & 0.83 \\
\hline & 3 & 1.27 & 0.06 & 1.30 & 1.10 & 0.27 & 0.92 \\
\hline & All & 1.12 & 0.20 & 0.82 & 1.11 & 0.60 & 0.05 \\
\hline \multirow{4}{*}{ 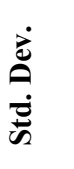 } & 1 & 0.99 & 0.36 & 1.20 & 0.56 & 0.55 & 0.29 \\
\hline & 2 & 0.82 & 0.99 & 0.09 & 0.28 & 1.59 & 1.58 \\
\hline & 3 & 0.99 & 0.21 & 1.14 & 0.88 & 0.07 & 0.78 \\
\hline & All & 0.88 & 0.22 & 0.62 & 0.49 & 0.43 & 0.78 \\
\hline
\end{tabular}

but between agitation and pre-agitation the effect size is not significant $(<0.5)$. Similarly, for PWD 2 the dissimilarity between the metrics for non-agitation and agitation epochs have insignificant effect size, and for PWD 3 insignificant effect size exists between agitation and pre-agitation epochs. From these observations, it can be inferred that the non-agitation epochs can be discriminated from both the pre-agitation and agitation epochs for PWD 1 and 3, but not for PWD 2. But even for PWD 1 and 3, the discrimination between pre-agitation and agitation epochs are not evident from the effect size analysis.

The outcome of the k-means clustering on the feature space provides a better perspective in discriminating the instances that consist the epochs. For the cluster of non-agitation instances, an average sensitivity of $93 \%$ is observed across all three PWD, whereas the average specificity is $25 \%$, for pre-agitation instances, the average sensitivity is $1 \%$ and the specificity is $99 \%$, and for agitation instances, sensitivity is $23 \%$ and specificity is $85 \%$. This result shows that unsupervised clusters of non-agitation instances contain lots of agitation and pre-agitation instances, i.e. most of the instances are clustered as non-agitation instances, and pre-agitation and agitation instances are rarely clustered in proper categories.

After conducting the PCA, we performed effect size analysis on the PCA scores from the first three principal components $(80 \%$ variance explained without standardization, 50\% explained with standardization) to separate among three categories of epochs. The result of the analyses is tabulated in Table 2. And the corresponding distribution plots are presented in Figures 6-9. It is notable that the mean metric indicates similar discrimination patterns for PWD 1 and 3, but for PWD 2, there appears high effect size in all three pairwise comparisons of epoch categories. This refers to the fact that PCA scores may be a better discriminator for PWD 2 .

The performance evaluation of the k-means clustering on PCA scores shows that the average sensitivity for clusters of nonagitation instances are reduced to $60 \%$ but specificity increases to $54 \%$. Similarly, for pre-agitation, the sensitivity increases to $7 \%$ and specificity decreases to $92 \%$. And for agitation instances, sensitivity raised to $41 \%$ and specificity reduced to $58 \%$. This result shows improvement in clustering of all three categories of instances. It indicates that further exploration of feature space may improve discrimination among the three categories of events.

\section{CONCLUSION}

In this work, we explore motion biomarkers that can be adopted to detect the early stages of an agitation episode in real time for
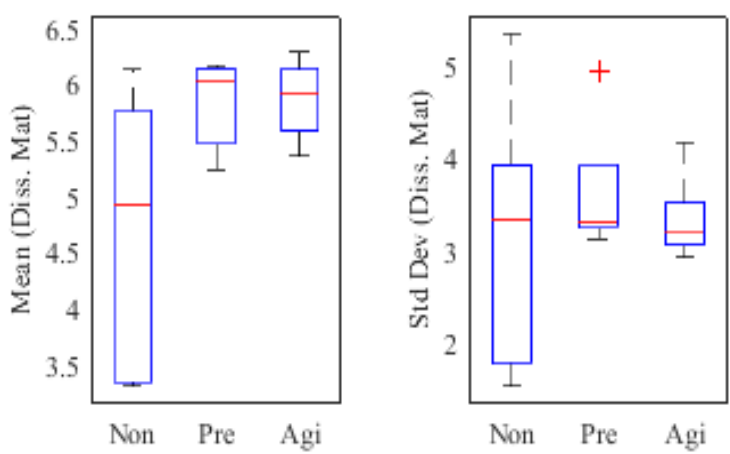

Figure 6. Distribution of mean and standard deviation of epoch PCA scores dissimilarity matrices for PWD 1.
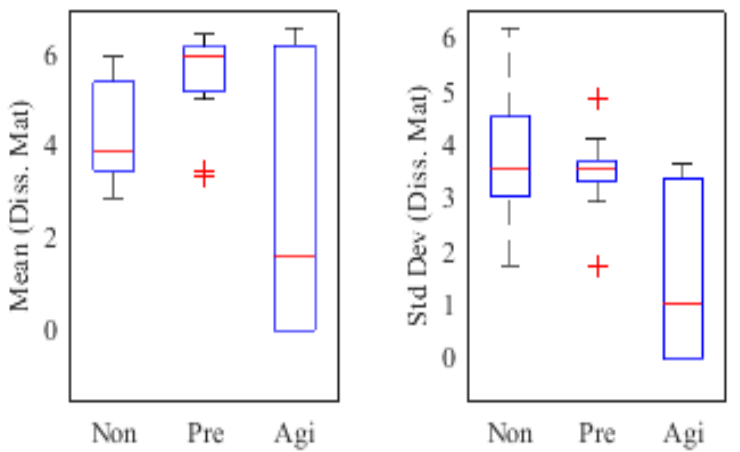

Figure 7. Distribution of mean and standard deviation of epoch PCA scores dissimilarity matrices for PWD 2.
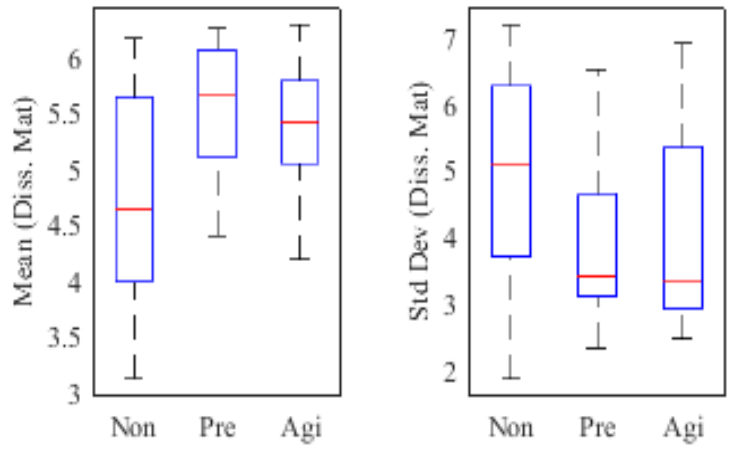

Figure 8. Distribution of mean and standard deviation of epoch PCA scores dissimilarity matrices for PWD 3.
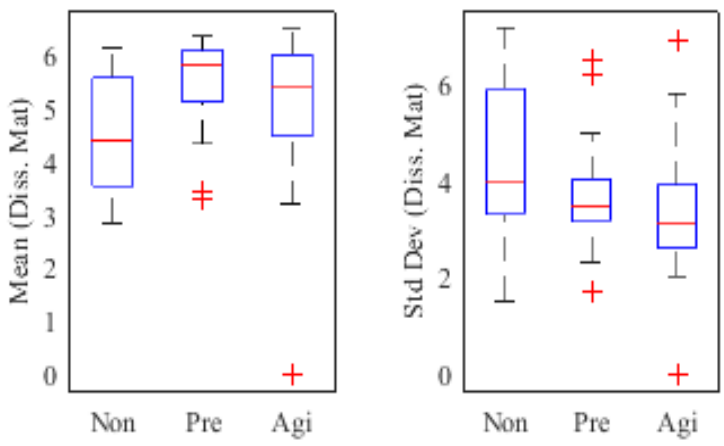

Figure 9. Distribution of mean and standard deviation of epoch PCA scores dissimilarity matrices for all PWDs. 
dementia patients. Toward that endeavor, we extract statistical, frequency, and energy features from the motion data of the PWD for representing the dynamic variation of the signals before and during the agitation compared to other periods. Preliminary result shows promising performance and potential of these features in differentiating the agitation, pre-agitation, and non-agitation periods. It is noteworthy that all data are acquired from real dementia population deployments as part of our BESI study [9].

We observe in this work that effect-size analysis and clustering do not perform similarly for all patients. Multiple factors can be attributed to this outcome including inter-caregiver rating variability and inter-person agitation pattern variability. Currently no established procedure exists for caregiver rating calibration, which is an important challenge for many health applications dependent on caregiver inputs. We attempted unsupervised clustering to mitigate those biases. We will focus on calibration agnostic feature selection as part of this ongoing exploration.

We note improvement in clustering performance after conducting PCA on the feature space. Though the changes were different for different subjects, this shows the potential of such exploration to find personalized biomarkers for agitation prediction. Other factors for personalization may include clinical assessments like CMAI scores and caregivers perceived severity levels. Also the performance of PCA based clustering shows the potential for further analysis of the feature space like discriminant analysis and factor analysis for better detection and prediction of agitation.

In this work, we only focused on stochastic parameters of the feature space. But temporal relationship among instances are an important aspect of time-series analysis, which we plan to incorporate in our next step. Other limitations of this study are due to the small sample size which is attributed to the difficulties in real patient recruitment and will be overcome with time.

To conclude, motion markers can be a useful predictor of escalation of dementia-related agitation. Our findings emphasize the potential for personalized models in this endeavor. Even with small sample size, we can provide beneficial support for the dyads. The success of this study will empower caregivers by providing just-in-time interventions and thus contribute in patient-caregiver welfare.

\section{REFERENCES}

[1] Alzheimer's Association. 2016 Alzheimer's disease facts and figures. Alzheimer's Dement (2016), 12(4):459-509.

[2] Colombo, M., Vitali, M., Cairati, R., et al. Behavioral and psychotic symptoms of dementia (BPSD) improvements in a special care unit: A factor analysis. Archives of Gerontology and Geriatrics (2007), 44(Sup):113-120.

[3] Sansoni, J., Anderson, K., Varona, L., et al. Caregivers of Alzheimer's patients and factors influencing institutionalization of loved ones: Some considerations on existing literature. Ann Ig (2013), 25(3):235-246.

[4] Cerejeira, J., Lagarto, L., and Ladinska, E.B. Behavioral and Psychological Symptoms of Dementia. Frontiers in Neurology 3 (2012): 73. PMC. Web. 15.

[5] Carrillo, M.C., Dishman, E., and Plowman, T. Everyday technologies for Alzheimer's disease care: Research findings, directions, and challenges. Alzheimer's \& Dementia 5.6 (2009): 479-488.
[6] Bharucha, A. J., et al. Intelligent assistive technology applications to dementia care: current capabilities, limitations, and future challenges. The American journal of geriatric psychiatry 17.2 (2009): 88-104.

[7] Moore, P., et al. Monitoring and detection of agitation in dementia: Towards real-time and big-data solutions. IEEE 3PGCIC (2013).

[8] Sacco, G., et al. Detection of activities of daily living impairment in Alzheimer's disease and mild cognitive impairment using information and communication technology. Clinical interventions in aging (2012), no. 7, pp. 539-549.

[9] Alam, R., et al. BESI: reliable and heterogeneous sensing and intervention for in-home health applications. IEEE CHASE 2017, In Press (2017).

[10] Morris, J. C. Clinical dementia rating: a reliable and valid diagnostic and staging measure for dementia of the Alzheimer type. International Psychogeriatrics 9. (1997): 173-176.

[11] Teng, E.L., and Helena, C.C. The Modified Mini-Mental State (3MS) examination. The Journal of clinical. psychiatry (1987).

[12] Nagels, G., et al. Actigraphic measurement of agitated behaviour in dementia. International journal of geriatric psychiatry 21.4 (2006): 388-393.

[13] Mahlberg, R., and Walther, S. Actigraphy in agitated patients with dementia. Zeitschrift für Gerontologie und Geriatrie 40.3 (2007): 178-184.

[14] David, R., et al. Ambulatory actigraphy correlates with apathy in mild Alzheimer's disease. Dementia 9.4: 509-516.

[15] Kirste, T., et al. Detecting the effect of Alzheimer's disease on everyday motion behavior. Journal of Alzheimer's Disease 38.1 (2014): 121-132.

[16] Li, Q., et al. Accurate, fast fall detection using gyroscopes and accelerometer-derived posture information. Wearable and Implantable Body Sensor Networks, (2009), BSN 2009.

[17] Kim, K.J., et al. Dementia wandering detection and activity recognition algorithm using tri-axial accelerometer sensors. Ubiquitous Information Technologies \& Applications, (2009), ICUT'09, IEEE, 2009.

[18] Bankole, A., et al. Continuous, non-invasive assessment of agitation in dementia using inertial body sensors. Wireless Health, ACM, (2011).

[19] Barth, A. T., et al. TEMPO 3.1: A body area sensor network platform for continuous movement assessment. BSN 2009.

[20] He, H., and Garcia, E.A. Learning from imbalanced data. IEEE Transactions on Knowledge and Data Engineering 21.9 (2009): 1263-1284.

[21] Xu, W., et al. Robust human activity and sensor location corecognition via sparse signal representation. IEEE Transactions on Biomedical Engineering 59, no. 11 (2012): 3169-3176. 